\title{
Study of pulmonary function tests in type 2 diabetes mellitus and their correlation with glycemic control and systemic inflammation
}

The authors declare no financial disclosure

\begin{abstract}
Introduction: Reduced lung function in diabetes has been described for long but its clinical importance is not yet clear. Also, limited literature is available regarding its association with sugar control, and its correlation with inflammatory markers. Thus, we aimed to study the pulmonary function test abnormalities and systemic inflammation in type 2 diabetes mellitus.

Material and methods: 100 patients with type 2 diabetes were divided into two groups depending on control of diabetes (group $\mathrm{A}-\mathrm{HbA1C} \leq 7 \%$ and group $\mathrm{B}-\mathrm{HbA} 1 \mathrm{C}>7 \%$ ). All the subjects selected underwent detailed evaluation including testing for $\mathrm{HbA} 1 \mathrm{C}$, HsCRP (high-sensitivity C-reactive protein), serum ferritin and serum fibrinogen, along with pulmonary function testing.

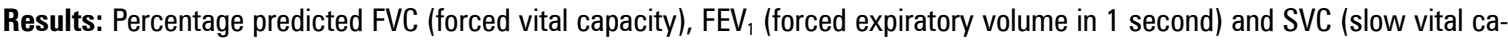
pacity) were significantly reduced, while mean values of inflammatory markers [fibrinogen $(p<0.001)$ and hsCRP $(p<0.002)$ ] were significantly higher in uncontrolled diabetes group. There was a significant negative correlation between $\mathrm{FEV}_{1}(r=-0.739, p$ $<0.001)$, FVC $(r=-0.370, p<0.001)$, SVC $(r=-0.635, p<0.001)$ with HbA1C. HbA1C had a positive correlation with hsCRP $(r=-0.308, p<0.002)$ and fibrinogen $(r=0.388, p<0.001)$.

Conclusion: Pulmonary functions were decreased and inflammatory markers like hsCRP, fibrinogen, and ferritin significantly increased in uncontrolled diabetics. Also, a potential association was seen between higher values of inflammatory markers like hsCRP and fibrinogen and decrease in lung function. This information, requiring confirmation with larger multicentre studies, remains important because of potential epidemiological, clinical and therapeutic implications.
\end{abstract}

Key words: diabetes, pulmonary function tests, inflammatory markers

Adv Respir Med. 2018; 86: 172-178

\section{Introduction}

Diabetes mellitus is a public health problem and has reached an epidemic proportion. Microvascular and macrovascular complications are the important cause of morbidity affecting various organ systems in diabetic patients. Several theories explain how glycosylation and oxidative stress leads to impaired collagen and elastin cross linkage with reduction in elasticity and strength of connective tissue which cause complications in diabetes. An extensive microvascular circulation and the abundant connective tissue in the lung raise the possibility that the lung may also be a target organ in diabetes [1]. Though an association of reduced lung function with diabetes has been described for many years, the clinical significance of this relationship is not yet known [2]. Some studies suggest that impaired lung function not only leads to increased respiratory morbidity, but also to adverse cardiovascular events and increased overall mortality [3, 4]. Systemic inflammatory activity also plays an important role in the pathogenesis of insulin resistance, and type 2 diabetes mellitus. It is likely that persistent inadequate glucose control over time may alter regulation of inflammatory pathways that are involved in the impairment of lung function [5]. 
It is still unknown if inadequate glucose control in type 2 diabetes is simultaneously associated with a decrease in lung function and increased systemic levels of inflammatory markers. In this study, we compare type 2 diabetes subjects basing on their glucose control profile (adequate vs. inadequate) and assess differences in lung functions (spirometry) and blood levels of the three known systemic inflammatory markers. Thus, the aims and objectives of the present study were the following: (1) to investigate the effect of type 2 diabetes mellitus on pulmonary function tests; (2) to correlate pulmonary function abnormalities with glycemic control; and (3) to study the association of pulmonary function abnormalities with systemic inflammation in type 2 diabetes mellitus.

\section{Material and methods}

This prospective study was conducted in the Department of Internal Medicine of a tertiary

- care teaching institute in 100 type 2 diabetic patients (aged 30 to 65)

- attending the institute after ethical clearance was obtained from institutional ethical

- committee. Inclusion and exclusion criteria of the study subjects are shown in Table 1.

Patients were classified into two groups

$\mathrm{A}$ and $\mathrm{B}$ depending on control of diabetes:

I. Group A -50 patients of type 2 diabetes with good control that is $\mathrm{HbA} 1 \mathrm{C} \leq 7 \%$.

II. Group B -50 patients of type 2 diabetes mellitus with poor glycemic control that is HbA1C $>7 \%$.

For all the subjects selected for the purpose of the study, parameters like age, height (m), we- ight (kg), body mass index (BMI) were measured. A structured pro forma was used to collect the relevant information regarding history, clinical findings and investigation test results of the patients.

All the subjects included in the study underwent following investigations:

1. Fasting blood sugar level, postprandial blood sugar level, HbA1C, HsCRP (high-sensitivity C-reactive protein, by Elisa method, quantitative), serum ferritin (Elisa method), Serum fibrinogen (Fibri-prest method).

2. Pulmonary function test (by MedisoftSpiroair). The spirometry report would take into account the predicted normal value for every subject.FVC (forced vital capacity), Forced expiratory volume in 1 second $\left(\mathrm{FEV}_{1}\right), \mathrm{FEV}_{1} /$ FVC, FEV ${ }_{1} \%\left(\mathrm{FEV}_{1}\right.$ as percentage of FVC), PEFR (peak expiratory flow rate), MMEF (maximum mid expiratory rate), SVC (slow vital capacity).

The statistical significance of categorical variables was determined by Chi-square test/ Fischer Exact Test for quantitative variable and unpaired 't' test/non-parametric Mann Whitney test. The correlation between PFT parameters and systemic inflammation was calculated by using Pearson Correlation Coefficient. Controlled data was analyzed with the same use of SPSS statistical software. Master chart showing the basic characters of the controlled diabetes group and uncontrolled group was prepared and rearranged as per the requirement to analyze the effect of HbA1C on PFT and inflammatory markers. The level of statistical significance was taken as $\mathrm{p}<0.05$.

\section{Table 1. Inclusion and Exclusion criteria of study population}

\begin{tabular}{ll}
\hline Inclusion Criteria & Exclusion Criteria \\
All patients with type 2 diabetic patients & Smokers \\
in the age group of 30 to 65 years be- & Pregnant females \\
longing to either sex attending OPD and & BMl $>30 \mathrm{~kg} / \mathrm{m}^{2}$ \\
admitted in medical wards & History of respiratory allergy and acute respiratory infection in the previous 3 months. Present \\
& or past history of respiratory disease that might affect lung function such as asthma, pleural \\
effusion /empyema, pulmonary tuberculosis, COPD, bronchiectasis, Interstitial lung disease, & lung cancer etc. \\
Known cardiovascular diseases like congestive cardiac failure, ischemic heart disease, diastolic \\
dysfunction \\
Previous diagnosis of rheumatoid arthritis or collagen vascular diseases, antibiotics use \\
in the last 4 weeks \\
Patients with chest deformities that would preclude conducting a reliable \\
a spirometric procedure \\
Patients with deranged renal function (serum creatinine $\geq 1.5 \mathrm{mg} / \mathrm{dl}$ ) \\
\hline
\end{tabular}




\section{Results}

We screened 110 subjects and 100 were finally included in the study (Table 2). The most frequent cause for exclusion was age (outside the stipulated inclusion criteria); past history of TB and smoking. Of the 100 studied type 2 diabetic subjects, 50 were classified as having uncontrolled diabetes (Group B, HbA1C > 7\%) and the remaining 50 were in controlled diabetes group (Group A, HbA1C < 7\%). The pulmonary function tests and blood investigations were conducted in 50 uncontrolled and 50 controlled diabetics.

\section{Baseline characteristics}

The baseline characteristics of both groups were compared (Supplementary File). There were no statistically significant differences in sex, age, height, weight, BMI. But there was significant difference between both the groups in duration of diabetes.

\section{Pulmonary function tests}

Various pulmonary function tests in diabetic patients are shown in Table 3. Percentage predicted FVC, $\mathrm{FEV}_{1}$ and SVC were significantly reduced in the uncontrolled diabetes group compared to the controlled ones (Fig. 1).

Table 2. Characteristics of diabetic patients at the baseline

\begin{tabular}{ll}
\hline Number of Subjects & 100 \\
Age (years) Range & 32 to 65 \\
Sex & 57 males and 43 females \\
& 50 uncontrolled type 2 DM, \\
Types of subjects & 50 controlled type $2 \mathrm{DM}$ \\
& $<5$ years: 22 patients \\
DM duration (years) & 5-10 years: 38 patients \\
& $>10$ years: 40 patients \\
HbA1C (Range) & 5.3 to $13.7 \%$ \\
\hline
\end{tabular}

Levels of inflammatory markers

The mean values of inflammatory markers, fibrinogen and hsCRP were higher in uncontrolled diabetes than in controlled diabetes and it was statistically significant for hsCRP $(p=0.002)$ and fibrinogen $(p<0.001)$. Serum ferritin though was discovered to be higher in uncontrolled diabetes group was found to be statistically insignificant $(\mathrm{p}$-value $=0.412)($ Table 4$)$.

\section{Correlation studies}

Following the above results, correlation was studied in details for $\mathrm{FEV}_{1}$, FVC, FEV $\mathrm{FV}_{1}$, SVC with glycemic control and inflammatory markers (Table 5). There was a negative correlation between $\mathrm{FEV}_{1}(\mathrm{r}=-0.739, \mathrm{p}<0.001), \mathrm{FVC}(\mathrm{r}=-0.370, \mathrm{p}<$ 0.001), SVC ( $r=-0.635, \mathrm{p}<0.001)$ with HbA1C which were statistically significant. $\mathrm{FEV}_{1} \%$ ( $\mathrm{r}=$ $0.107, p=0.291$ ) ratio had statistically insignificant correlation with HbA1C. Thus it implies that as the $\mathrm{HbA} 1 \mathrm{C}$ value rises there is a decrease in $\mathrm{FEV}_{1}$, FVC and SVC values.

With respect to correlation of glycemic control with inflammatory markers, HbA1C has a positive correlation with hsCRP $(\mathrm{r}=-0.308$, $\mathrm{p}=0.002)$ and fibrinogen $(\mathrm{r}=0.388, \mathrm{p}=0.001)$ which were statistically significant and statistically insignificant correlation with ferritin( $\mathrm{r}=$ 0.076, p < 0.453) (Table 5). Thus as the HbA1C rises there is significant increase in levels of hsCRP and fibrinogen but not in ferritin levels.

There was a negative correlation of FVC, $\mathrm{FEV}_{1}$, and SVC with hsCRP and fibrinogen which was statistically significant. There was statistically insignificant correlation of $\mathrm{FVC}, \mathrm{FEV}_{1}$, and SVC with ferritin (Table 6). The $\mathrm{FEV}_{1} \%$ had a statistically insignificant correlation with hsCRP, ferritin, and fibrinogen. Thus, as FVC, $\mathrm{FEV}_{1}$, SVC decreased, there was an associated increase in inflammatory markers like hsCRP and fibrinogen concentration.

Table 3. Summary of pulmonary function tests in study groups

\begin{tabular}{lccc}
\hline Variable & $\begin{array}{c}\text { Controlled diabetes group } \\
(\mathbf{n}=\mathbf{5 0}) \\
(\mathbf{m e a n} \pm \mathbf{S D})\end{array}$ & $\begin{array}{c}\text { Uncontrolled diabetes group } \\
\text { ( } \mathbf{n = 5 0 )}\end{array}$ & $\begin{array}{c}\text { p-value } \\
\text { (mean } \pm \mathbf{~ S D )}\end{array}$ \\
\hline Percentage predicted FEV 1 & $100.1 \pm 21.8$ & $80.27 \pm 22.5$ & $<0.001$ \\
Percentage predicted FVC & $101.95 \pm 18.1$ & $69.36 \pm 15.34$ & $<0.001$ \\
Percentage predicted FEV $\%$ & $97.97 \pm 12.5$ & $99.27 \pm 12.9$ & 0.612 \\
Percentage predicted PEFR & $115.48 \pm 63.49$ & $91.14 \pm 46.46$ & 0.031 \\
Percentage predicted SVC & $83.62 \pm 14.82$ & $63.99 \pm 13.31$ & $<0.001$ \\
Percentage predicted MMEF & $86.72 \pm 16.50$ & $82.85 \pm 12.54$ & 0.095 \\
\hline
\end{tabular}




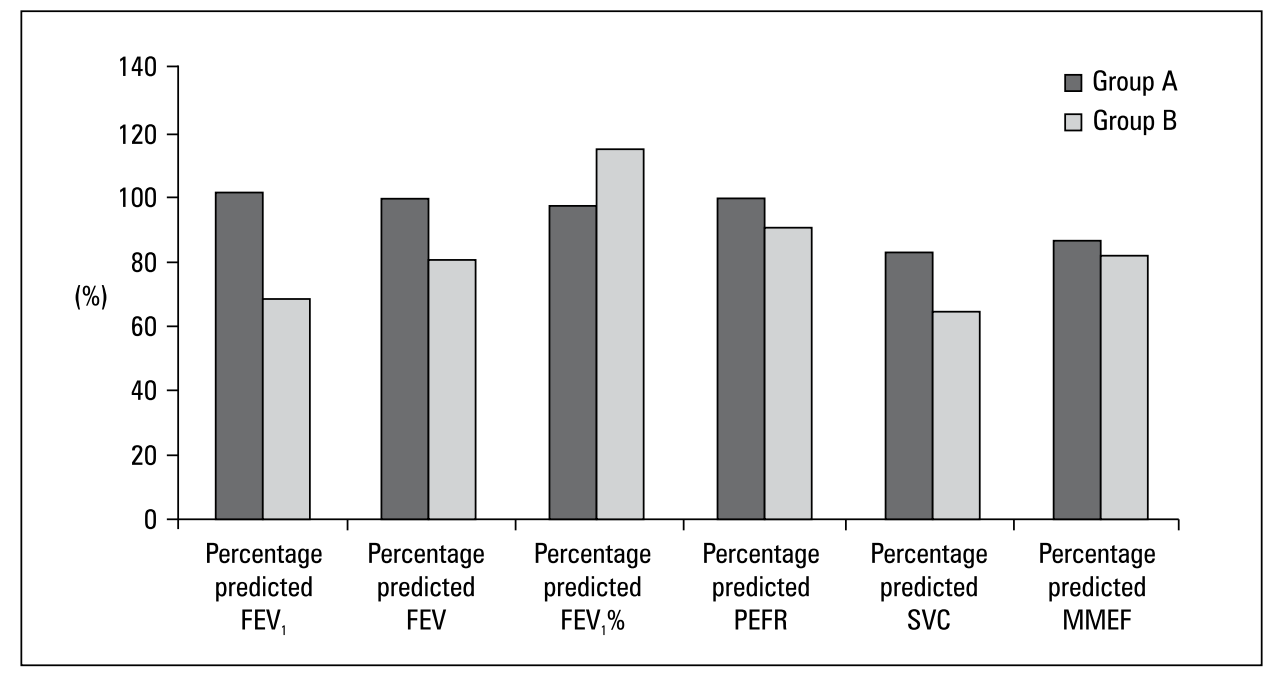

Figure 1. Mean values of Pulmonary Function tests in both study groups (controlled vs uncontrolled diabetic subjects); abbreviations in the text

Table 4. Summary of inflammatory markers in both groups

\begin{tabular}{lccc}
\hline Marker & $\begin{array}{c}\text { Controlled diabetes group } \\
(\mathbf{n}=\mathbf{5 0 )} \\
(\mathbf{m e a n} \pm \mathbf{S D})\end{array}$ & $\begin{array}{c}\text { Uncontrolled diabetes group } \\
(\mathbf{n}=\mathbf{5 0 )} \\
(\text { mean } \pm \text { SD) }\end{array}$ & p-palue \\
\hline hsCRP $(\mathrm{mg} / \mathrm{l})$ & $2.5 \pm 1.1$ & $3.24 \pm 1.08$ & 0.002 \\
Ferritin (ug/l) & $93.09 \pm 44.8$ & $96.72 \pm 34.28$ & 0.412 \\
Fibrinogen $(\mathrm{mg} / \mathrm{dl})$ & $295.02 \pm 31.27$ & $324.22 \pm 41.56$ & $<0.001$ \\
\hline
\end{tabular}

Table 5. Correlation of glycemic control with pulmonary function tests and inflammatory markers in diabetes patients

\begin{tabular}{lccc}
\hline Glycemic control & Pulmonary function tests & Pearson correlation & p-value \\
\hline \multirow{3}{*}{$\mathrm{HbA1C}^{*}$} & $\mathrm{FEV}_{1}$ & -0.739 & $<0.001$ \\
& $\mathrm{FVC}$ & -0.370 & $<0.001$ \\
& $\mathrm{FEV}_{1} \%$ & 0.107 & 0.291 \\
& $\mathrm{SVC}$ & -0.635 & $<0.001$ \\
\hline $\mathrm{HbA1C}$ & Inflammatory markers & Pearson correlation & p-value \\
& hsCRP & 0.308 & 0.002 \\
& Ferritin & 0.076 & 0.453 \\
& Fibrinogen & 0.388 & 0.001 \\
\hline
\end{tabular}

\section{Discussion}

India is one of the two major global hubs for the rapidly escalating type 2 diabetes epidemic, along with China. Till now pulmonary complications of diabetes were least studied and the exact etiology of pulmonary complication is still not known. So far most of the studies come from western world and only few investigations have been conducted in India regarding pulmonary complications of type 2 DM [6-10].
In the present study one hundred examined type 2 diabetes patients were divided in two groups, controlled diabetes group and uncontrolled diabetes group. The basic characteristics like age, gender, height, weight, BMI in our study groups were analyzed and were found to be comparable. Respiratory parameters such as percentage predicted FVC, FEV ${ }_{1}, \mathrm{FEV}_{1} \%$, SVC, MMEF, and PEFR were recorded in both the groups. Percentage predicted FVC, $\mathrm{FEV}_{1}$ and SVC were significantly reduced in the uncontrolled diabetes group com- 


\section{Table 6. Correlation of pulmonary function tests (PFTs)} and inflammatory markers

\begin{tabular}{cccc}
\hline PFTs & $\begin{array}{c}\text { Inflammatory } \\
\text { markers }\end{array}$ & $\begin{array}{c}\text { Pearson } \\
\text { correlation } \\
\text { r value }\end{array}$ & p-value \\
\hline \multirow{3}{*}{ FVC } & hsCRP & -0.393 & $<0.001$ \\
& Ferritin & -0.105 & 0.297 \\
& Fibrinogen & -0.550 & $<0.001$ \\
FEV $_{1}$ & hsCRP & -0.310 & $<0.002$ \\
& Ferritin & 0.012 & 0.905 \\
& Fibrinogen & -0.47 & $<0.001$ \\
FEV $_{1} \%$ & hsCRP & -0.166 & 0.100 \\
& Ferritin & -0.073 & 0.472 \\
SVC & Fibrinogen & -0.73 & 0.471 \\
& hsCRP & -0.377 & $<0.001$ \\
& Ferritin & -0.053 & 0.60 \\
& Fibrinogen & -0.397 & $<0.001$ \\
\hline
\end{tabular}

Abbreviations in the text

pared to the controlled diabetes group. Percentage predicted MMEF was mildly lowered in both the groups but the reduction was not statistically significant ( $\mathrm{p}$-value $<0.095)$. Percentage predicted $\mathrm{FEV}_{1} \%$ was greater in the uncontrolled group but the increase was statistically insignificant (p-value $<0.612$ ). A reduction in vital capacity as evidenced by a fall in SVC, FVC and FEV , $_{\text {, }}$ with normal $\mathrm{FEV}_{1} \%$ is suggestive of a tendency towards a restrictive ventilatory pattern. Percentage predicted PEFR was also reduced in both the groups that is controlled and uncontrolled diabetes groups but reduction was greater in the uncontrolled group and the decrease was statistically significant ( $p$-value $<0.031$ ). As the observations made above are indicative of primarily restrictive ventilatory pattern of lung disorder and in this group of disorder PEFR and MMEF have an insignificant role. On correlating pulmonary functions with glycemic control we found that there was a negative correlation between $\mathrm{FEV}_{1}$ $(\mathrm{r}=-0.739, \mathrm{p}<0.001), \mathrm{FVC}(\mathrm{r}=-0.370, \mathrm{p}<0.001)$ and SVC $(r=-0.635, p<0.001)$ and HbA1C and it was statistically significant. This indicates that as the glycemic control worsens the pulmonary functions decline. $\mathrm{FEV}_{1} / \mathrm{FVC}(\mathrm{r}=0.107, \mathrm{p} 0.291)$ ratio had insignificant correlation with HbA1C.

Our findings were in accordance with previous studies. Framingham Heart study by Walter etal. [11], demonstrated reduction of FVC, $\mathrm{FEV}_{1}$ and an increase in $\mathrm{FEV}_{1} / \mathrm{FVC}$ ratio in diabetic patients suggesting a restrictive pattern of ventilatory impairment]. The lung functions decreased as the serum glucose concentration increased. In another study by Davis et al. [12], it was demonstrated that mean percentage predicted values of each spirometric measure decreased by $10 \%$ in the whole cohort at baseline and absolute measures continued to decline at an annual rate of 68,71 , and $84 \mathrm{ml} /$ year and $17 \mathrm{l} / \mathrm{min}$ for FVC, $\mathrm{FEV}_{1}$, VC, and PEF respectively, in the 125 prospectively studied patients. Declining lung function measures were consistently predicted by poor glycemic control in the form of a higher updated mean HbA1C, follow-up HbA1C, or follow-up fasting plasma glucose. Keerthi et al. [6] studied 50 type 2 diabetes mellitus patients and 50 healthy controls in India and found decrease in pulmonary function tests in diabetics as compared to nondiabetics. They also found that females were more prone to respiratory dysfunction than male diabetics. Study by Verma et al. [7] also showed restrictive type of pulmonary ventilatory impairment as evidenced by significant reduction in $\mathrm{FVC}, \mathrm{FEV}_{1}$, and normal $\mathrm{FEV}_{1} / \mathrm{FVC}$ in type-2 diabetes patients.

The diabetic lung is best characterized as a loss of physiological reserves. There are different hypotheses that explain the reduced spirometric measurements in diabetics. Preliminary reports of histopathologic changes in the lungs of diabetic patients reveal basal lamina thickening and fibrosis $[13,14]$. In both human and animal studies, diabetic lungs have demonstrated diabetic microangiopathy of the alveolar septal capillaries, with a thickened epithelial and capillary basement membrane, and increased extracellular matrix and connective tissue [15]. In diabetic patients, chronic hyperglycemia can bring about a rise in collagen molecule synthesis and cross-linking via the acceleration of advanced glycation end-products, which can also negatively influence lung function [16, 17].

Despite the unclear nature of the direction of association between diabetes and lung dysfunction, this relationship remains important because of potential epidemiological, clinical and therapeutic implications. Although the magnitude of lung function impairment secondary to diabetes is likely to cause only subclinical abnormalities, a loss of pulmonary reserve capacity may become clinically important in the context of superimposed conditions, such as age-related lung function decline, obesity, smoking, hypoxia associated with acute or chronic disease or fluid overload secondary to cardiac or renal failures which are common complications of diabetes [18]. Similarly, the impairment in lung function seen in patients with diabetes can lower the threshold for clinical manifestations of acute or chronic lung diseases. 
In a study by Ehrlich et al. [19], it was reported that diabetics are at increased risk of several pulmonary condititions (asthma, COPD, fibrosis and pneumonia) but not lung cancer and this may be due to declining lung function.

Inflammatory markers in diabetes: The levels of hsCRP were higher in the controlled diabetes than the concentration of hsCRP in the controlled group with p-value of 0.002 . Thus, there is a significant increase in hsCRP in the uncontrolled diabetes group. The study group was further divided into high risk and moderate and low risk patients basing on their hsCRP levels. Among the study group 13 subjects in the controlled diabetes group and 38 from uncontrolled diabetes group fell into high risk (hsCRP $>3 \mathrm{mg} / \mathrm{dl}$ ) category. Thus there is a statistically significant increase in the number of high risk patients in the uncontrolled diabetes group $(p=0.002)$ There was also a statistically significant increase in the levels of fibrinogen in the uncontrolled diabetes group compared to controlled diabetes with a p-value of $<0.001$.

There was an increase in the levels of ferritin in uncontrolled group compared to the controlled group but the difference was not statistically significant ( $p$-value $=0.412)$. Since ferritin levels are known to be different in two genders i.e. males and females, both the groups were further subdivided into males and females and were compared. It was found that ferritin levels were significantly raised in females in the uncontrolled group in comparison to the controlled group $(p=0.005)$ whereas the raise was not statistically significant in males $(\mathrm{p}=0.780)$.

In our study HbA1C had a positive correlation with hsCRP and fibrinogen which was statistically significant. HbA1C had an insignificant correlation with ferritin. Thus there is a significant increase in inflammatory markers like hsCRP and fibrinogen as the HbA1C rises. Various studies in the past have found association between inflammation and diabetes [3, 5]. Pitsavos et al. [20] in the ATTICA study support a positive association between low-grade inflammation (TNF alpha and hsCRP) and diabetes in a population-based sample of men and women without any evidence of cardiovascular disease.

Correlation of lung functions with inflammatory markers: There was a negative correlation of FVC, $\mathrm{FEV}_{1}$, and SVC with hsCRP and fibrinogen which was statistically significant. There was statistically insignificant correlation of FVC, $\mathrm{FEV}_{1}$, and SVC with ferritin. The $\mathrm{FEV}_{1} \%$ had a statistically insignificant correlation with hsCRP, ferritin, and fibrinogen. Thus from the above results it can be concluded that as $\mathrm{FVC}, \mathrm{FEV}_{1}$, SVC decreases there is an associated increase in inflammatory markers like hsCRP, fibrinogen levels. In summary, subjects with uncontrolled diabetes also had significantly higher levels of inflammatory markers (fibrinogen, and hsCRP), along with decreased lung functions.

Dennis et al in their study involving diabetic patients concluded that diabetic subjects with inadequate control had lower FVC and $\mathrm{FEV}_{1}$ than those with adequate control and also had significantly higher levels of inflammatory mediators like ferritin, hsCRP, fibrinogen and TNF- $\alpha$ suggesting a potential association [5]. Though our study had similar findings with hsCRP and fibrinogen, there was no significant correlation with serum ferritin and lung dysfunction.

Limitation of our study was its cross sectional hence only one measurement of $\mathrm{HbA1C}$ was done to ascertain glycemic control restricting its usefulness in labelling disease control. In addition, PFT was limited to spirometry alone.

\section{Conclusions}

To conclude, our findings not only have confirmed that diabetic subjects show reduction in pulmonary functions which is higher in patients with inadequate glucose control but also have showed that Indian diabetic subjects with inadequate control also had significantly higher levels of inflammation markers (fibrinogen and hsCRP). There was also an increase in inflammatory markers like hsCRP and fibrinogen with decrease in lung function suggesting a potential association. Our results need to be confirmed by longitudinal studies (given that diabetic control is a time-dependent variable). Detailed lung functions including diffusion studies can be investigated in future for better understanding of the diabetic lung dysfunction. Hopefully, it can also be shown that better glucose control can result not only in lack of further decrease in lung function but also in attenuation of the inflammatory response. Our findings, however, should make clinicians more aware of this association and add to the necessity of advising diabetic patients of the need for adequate glucose control.

\section{Conflict of interest}

The authors declare no conflict of interest.

\section{References:}

1. Sandler M. Is the lung a 'target organ' in diabetes mellitus? Arch Intern Med. 1990; 150(7): 1385-1388, indexed in Pubmed: 2196023. 
2. Kaparianos A, Argyropoulou E, Sampsonas F, et al. Pulmonary complications in diabetes mellitus. Chron Respir Dis. 2008; 5(2): 101-108, doi: 10.1177/1479972307086313, indexed in Pubmed: 18539724.

3. Lim SY, Rhee EJ, Sung KC. Metabolic syndrome, insulin resistance and systemic inflammation as risk factors for reduced lung function in Korean nonsmoking males. J Korean Med Sci. 2010; 25(10): 1480-1486, doi: 10.3346/jkms.2010.25.10.1480, indexed in Pubmed: 20890430.

4. Sampol G, Lecube A. Type 2 diabetes and the lung: a bidirectional relationship. Endocrinol Nutr. 2012; 59(2): 95-97, doi: 10.1016/j.endonu.2011.12.003, indexed in Pubmed: 22265762.

5. Dennis RJ, Maldonado D, Rojas MX, et al. Inadequate glucose control in type 2 diabetes is associated with impaired lung function and systemic inflammation: a cross-sectional study. BMC Pulm Med. 2010; 10: 38, doi: 10.1186/1471-2466-10-38, indexed in Pubmed: 20659337.

6. G SK. Deterioration of pulmonary functions in type 2 diabetes mellitus. IOSR Journal of Pharmacy and Biological Sciences. 2012; 1(1): 39-43, doi: 10.9790/3008-0113943.

7. Verma S, Goni M, Kudyar RP. Assesment of pulmonary function tests in patients with diabetes mellitus. JK Science. 2009; 11(2): 71-74.

8. Kanyakumari DH, Natraj SM, et al. correlation of duration of diabetes and pulmonary function test in type 2 diabetes mellitus patients. Int. J. Biol Med Res. 2011; 28(2): 89-92.

9. Shah SH, Sonawane P, Nahar P, et al. Pulmonary function tests in type 2 diabetes mellitus and their association with glycemic control and duration of the disease. Lung India. 2013; 30(2): 108-112, doi: 10.4103/0970-2113.110417, indexed in Pubmed: 23741090 .

10. Dharwadkar AR, Dharwadkar AA, Banu G, et al. Reduction in lung functions in type-2 diabetes in Indian population: correlation with glycemic status. Indian J Physiol Pharmacol. 2011; 55(2): 170-175, indexed in Pubmed: 22319899.

11. Walter RE, Beiser A, Givelber RJ, et al. Association between glycemic state and lung function: the Framingham Heart Stu- dy. Am J Respir Crit Care Med. 2003; 167(6): 911-916, doi: 10.1164/rccm.2203022, indexed in Pubmed: 12623860.

12. Davis WA, Knuiman M, Kendall P, et al. Fremantle Diabetes Study. Glycemic exposure is associated with reduced pulmonary function in type 2 diabetes: the Fremantle Diabetes Study. Diabetes Care. 2004; 27(3): 752-757, indexed in Pubmed: 14988297.

13. Irfan M, Jabbar A, Haque AS, et al. Pulmonary functions in patients with diabetes mellitus. Lung India. 2011; 28(2): 89-92, doi: 10.4103/0970-2113.80314, indexed in Pubmed: 21712938

14. Weynand B, Jonckheere A, Frans A, et al. Diabetes mellitus induces a thickening of the pulmonary basal lamina. Respiration. 1999; 66(1): 14-19, doi: 10.1159/000029331, indexed in Pubmed: 9973685.

15. Ljubić S, Metelko Z, Car N, et al. Reduction of diffusion capacity for carbon monoxide in diabetic patients. Chest. 1998; 114(4): 1033-1035, indexed in Pubmed: 9792573.

16. Goldman MD. Clinical application of forced oscillation. Pulm Pharmacol Ther. 2001; 14(5): 341-350, doi: 10.1006/ pupt.2001.0310, indexed in Pubmed: 11603948.

17. Sandler M, Bunn AE, Stewart RI. Cross-section study of pulmonary function in patients with insulin-dependent diabetes mellitus. Am Rev Respir Dis. 1987; 135(1): 223-229, doi: 10.1164/arrd.1987.135.1.223, indexed in Pubmed: 3492160.

18. Hsia CCW, Raskin P. Lung function changes related to diabetes mellitus. Diabetes Technol Ther. 2007; 9 Suppl 1: S73-S82, doi: 10.1089/dia.2007.0227, indexed in Pubmed: 17563307.

19. Ehrilch SF, Quesenberry CP, Van Den Eden SK, et al. Patients dignosed with diabetes are at increased risk for asthama, chronic obstructive pulmonary disease, pulmonary fibrosis, and pneumonia but not lung cancer. Diabetes Care. 2010; 33(1): 55-60.

20. Pitsavos C, Tampourlou M, Panagiotakos DB, et al. Association Between Low-Grade Systemic Inflammation and Type 2 Diabetes Mellitus Among Men and Women from the ATTICA Study. Rev Diabet Stud. 2007; 4(2): 98-104, doi: 10.1900/ RDS.2007.4.98, indexed in Pubmed: 17823694. 